\section{Spontaneous Axillary Cystic Hygroma: A Rare Entity in Adults}

Sir,

A lymphocele or cystic hygroma is a benign congenital lymphatic malformation that occurs due to incomplete development of lymphatics, commonly in infants. ${ }^{1}$ Approximately, $90 \%$ of cystic hygromas are identified before the age of 2 years. They occur frequently in cervical, axillary, inguinal, retroperitoneal, and thoracic regions. ${ }^{2}$

A 30-year woman presented with a lump in her right axilla for last 2 months, which was gradually increasing in size. She was, otherwise, completely asymptomatic.

On examination, there was a $10 \times 6 \mathrm{~cm}$, soft, fluctuant, non-tender swelling in the right axilla with normal overlying skin (Figure 1).

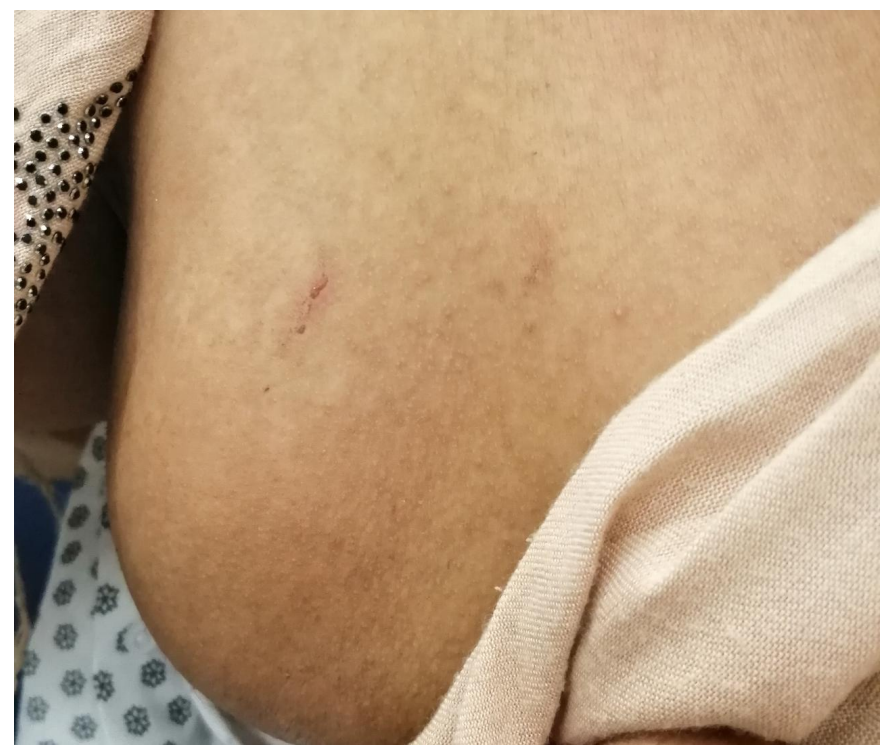

Figure 1: An ill-defined mass lesion in the right axilla. The overlying skin is normal.

Breast examination revealed no mass and no lymphadenopathy. Chest radiography was unremarkable. Ultrasound showed a well circumscribed complex collection of $4 \times 7.1 \times 11$ $\mathrm{cm}$ in right axilla and normal breast. Fine needle aspiration was carried out and $50 \mathrm{~mL}$ of straw-colour translucent fluid was aspirated. The cyst aspirate fluid was assessed by Gram staining and Ziehl-Neelsen staining. Microscopy and culture for routine organisms and tuberculosis bacilli, showed no abnormality. Patient was followed-up in OPD.

Patient again presented with swelling and pain. The cyst enlarged in size over two weeks. Ultrasound was carried out and showed a well-defined cyst with evidence of septations. We planned for excision of cyst.
Thus, we made a transverse incision in right axilla. The cyst was dissected away from major axillary vessels (Figure 2). The wound was closed primarily after keeping drain inside. Postoperative recovery was uneventful.

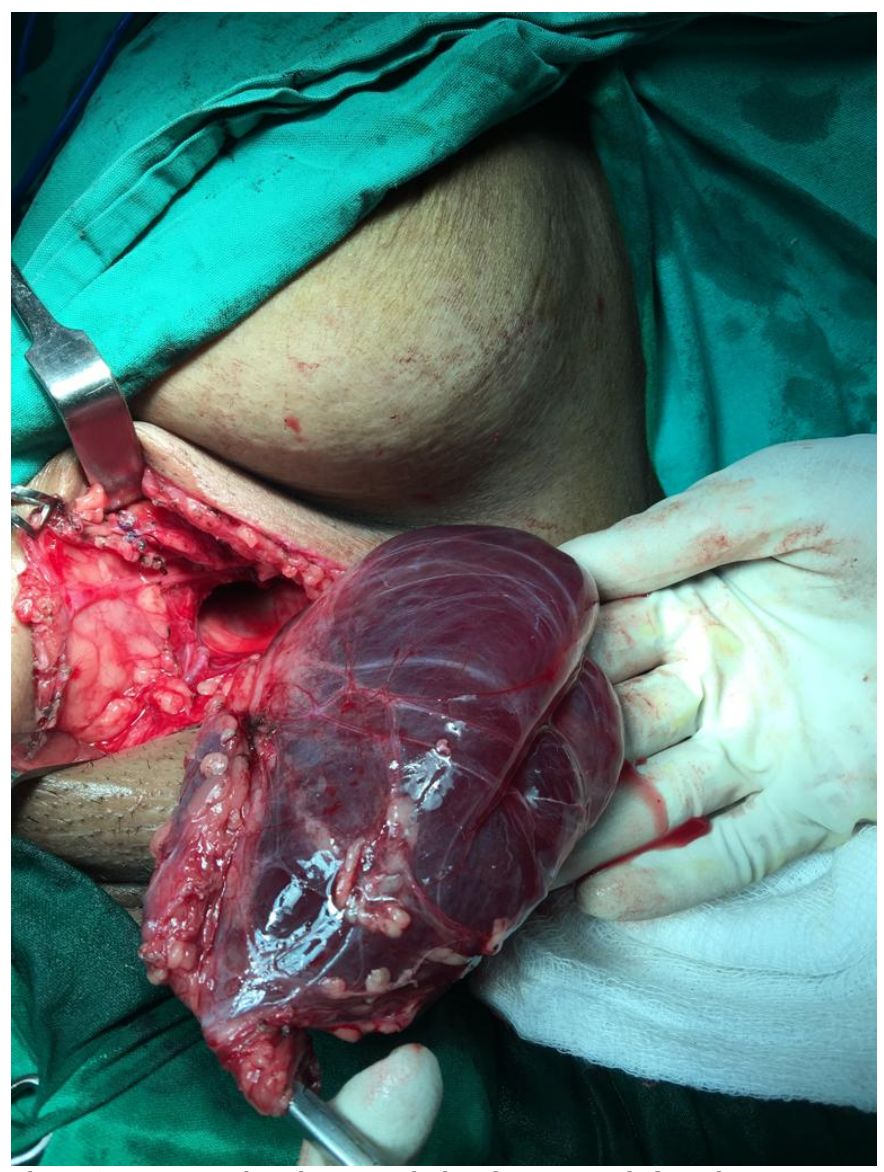

Figure 2: Peroperative photograph showing removal of cyst in toto.

On histopathological evaluation, the specimen measured $8 \times 4$ $\mathrm{cm}$. The average wall thickness was $0.7 \mathrm{~cm}$. No focal solid areas were seen. There were some areas of hemorrhage on the wall with brown patches. On microscopy, sections showed lymphoid tissue with intact architecture, along with adipose tissue, exhibiting lesion composed of thin walled vessels containing proteinaceous material and few scattered lymphocytes. No red blood cells were seen. Local fibroplasia was present with granulation tissue. There were no signs of granulomatous infection, atypia ormalignancy.

Our case is unusual as it presented as a large de-novo axillary lymphocele or cystic hygroma, with no history of trauma. Smith et al. classified lymphatic malformations into microcystic $(<2 \mathrm{~cm})$ and macrocystic $(>2 \mathrm{~cm}) .{ }^{3}$ Treatment of a cystic hygroma has historically required surgical excision which some believe to be preferred in adults as lesions are better circumscribed. ${ }^{4}$ Excision was successful after first failed attempt of aspiration. Several case reports have demonstrated the inefficacy of simple aspiration or antibiotics. ${ }^{5}$ Longterm follow-up has been suggested as an important element of 
management, as recurrence has occurred as late as 6 years after removal.

\section{CONFLICT OF INTEREST:}

Authors declared no conflict of interest.

\section{AUTHORS' CONTRIBUTION:}

HK: Drafting, designand literature review.

ASM: Supervision.

MRA: Final approval.

KAS: Final drafting.

SS: Critical revision.

RN: Literature review.

\section{REFERENCES}

1. Bhavsar DT, Saeed-Vafa S. Harbison S. Inniss. Retroperitoneal cystic lymphangioma in an adult: A case report and review of the literature. World J Gastrointest Pathophysiol 2010; 1:171-6.

2. Veeraraghavan CG. Denny A. Lingappa. Cystic hygroma in an adult; A case report. Libyan J Med 2009; 4:160-1.

3. Smith RJ, Burke DK, Sato Y, Poust RI, Kimura K, Bauman NM. OK-432 therapy for lymphangiomas. Arch Otolaryngol
Head Neck Surg 1996; 122(11):1195-9.

4. Mulliken JB, Glowacki J. Hemangiomas and vascular malformations in infants and children: A classification based on endothelial characteristics. Plast Reconstr Surg 1982; 69(3):412-22.

5. Gow L, Gulati R, Khan A, Mihaimeed F. Adult-onset cystic hygroma: A case report and review of management. Grand Rounds 2011; 11(1):5-11.

Hina Khan, Amjad Siraj Memon, Mujeeb ur Rehman Abbasi, Khursheed Ahmed Samo, Summaya Saeed and Rubab Nafees General Surgical Unit 3, Dr Ruth KM Pfau, Civil Hospital Karachi, Pakistan

Correspondence to: Dr. Hina Khan, General Surgical Unit 3, Dr Ruth KM Pfau, Civil Hospital Karachi, Pakistan E-mail: hina.khan@duhs.edu.pk

Received: August 05, 2019; Revised: August 05, 2019; Accepted: August 27, 2019

DOI: https://doi.org/10.29271/jcpsp.2020.07.769 